\title{
Prevention of Invasive Meningococcal Disease in the United States: Current State of the Art
}

\author{
Corwin A Robertson $^{1 *}$, Philipp Oster ${ }^{2}$, David R Johnson ${ }^{1}$, Albert Reinhardt ${ }^{1}$, David P Greenberg ${ }^{1}$ and Michael D Decker $^{1}$
}

${ }^{1}$ Sanofi Pasteur, Swiftwater, Pennsylvania, USA

${ }^{2}$ Sanofi Pasteur, Lyon, France

\begin{abstract}
Neisseria meningitidis causes potentially fatal and often debilitating invasive meningococcal disease (IMD). IMD remains a serious health problem for healthcare providers and public health officials despite approximately $80 \%$ of US IMD being vaccine-preventable. In 2005, the Advisory Committee on Immunization Practices (ACIP) recommended routine immunization of adolescents aged 11-12 years and, in 2007, this recommendation was expanded to include all adolescents aged 11-18 years. Adolescent disease burden has since declined. For new vaccination strategies to improve beyond standard of care, they must account for current epidemiologic trends. This article reviews the current epidemiology of IMD in the United States, highlighting known risk factors. Prior ACIP recommendations (and their rationale) since the approval of the first quadrivalent conjugate meningococcal vaccine in 2005 are summarized. The scientific and logistical issues associated with extending those recommendations to include infants and other at-risk groups are also discussed.
\end{abstract}

Keywords: Meningococcal disease; Immunization; Conjugate vaccine; Adolescents; Infants, epidemiology; United states; Advisory committee on immunization practices

Abbreviations: ABCs: Active Bacterial Core surveillance; ACIP: Advisory Committee on Immunization Practices; FDA: US Food and Drug Administration; HIV: Human Immunodeficiency Virus; hSBA: Serum Bactericidal Assay using Human Complement; IMD: Invasive Meningococcal Disease; MCC: Meningococcal Serogroup C Conjugate Vaccine; MCV4: Quadrivalent Conjugated Meningococcal Vaccine; MPSV4: Meningococcal Quadrivalent Polysaccharide Vaccine; OMV: Outer Membrane Vesicles; rSBA: Serum Bactericidal Assay using Baby Rabbit Complement; VE: Vaccine Effectiveness

\section{Introduction}

Invasive Meningococcal Disease (IMD) remains a vexing problem for providers, public health officials and advisory bodies, despite multiple healthcare intervention strategies. This review examines immunization strategies for IMD in light of recent US epidemiologic trends. Future vaccines and vaccination strategies are also considered.

Neisseria meningitidis is an aerobic gram-negative bacterium responsible for IMD; there are at least a dozen phenotypically distinct $N$. meningitidis serogroups. These serogroups are differentiated by the biochemical and structural characteristics of the bacterium's polysaccharide capsule. The meningococcal polysaccharide capsule plays a major role in determining the characteristics of the bacterium. Five serogroups-A, B, C, W (formerly W-135) and Y-are responsible for the majority of invasive disease [1]. In the United States, serogroups $\mathrm{B}, \mathrm{C}$ and $\mathrm{Y}$ are major causes of IMD, with each responsible for approximately one third of cases [2]. The proportion of IMD cases caused by each serogroup varies over time and by age group. For children aged 1 year and younger, more than 50\% of IMD cases are caused by serogroup B, for which there currently is no vaccine licensed in the United States [3]. By contrast, 75\% of IMD cases among patients aged 11 years and older are vaccine-preventable, caused by serogroups C, W, or Y [3].

The most common clinical manifestations of IMD are meningitis, septicemia and pneumonia [4]. Although less common than streptococcal and pneumococcal disease [5-8], IMD is associated with high morbidity and mortality, and approximately 11-19\% of individuals with the condition develop permanent physical or neurologic sequelae [9]. In one US study examining data collected during 1998-2007, IMD was associated with an overall case-fatality rate of $10.1 \%$ [10]. Among college students aged 17-4 years in Pennsylvania, a case-fatality rate of $19 \%$ was observed [11]. With meningococcemia, the risk is even greater, with up to $40 \%$ of cases ending in death [4]. Of particular concern is the rapid course of the disease, with the possibility of death occurring in as few as 24 hours [11].

The epidemiology and incidence of IMD can vary dramatically, both over time and geographically $[4,12]$. Since 2000, the incidence of IMD has decreased in the United States and is currently at an historic low. Yet, some patient populations remain at elevated risk for disease. These at-risk populations include infants and very young children, adolescents, the elderly [13] and individuals with compromised immune systems or certain genetic polymorphisms [14]. Other factors (e.g., environmental and socioeconomic) are also associated with an increased risk of disease and include residing in a crowded setting, such as a dormitory, and smoking or exposure to second-hand smoke [15].

Vaccines against selected meningococcal serogroups are available and can prevent a substantial percentage of IMD cases. Furthermore, these vaccines can be used in a wide range of patient populations. In the United States, three quadrivalent vaccines developed against serogroups A, C, W and Y are currently available [4]. The first quadrivalent vaccine was meningococcal quadrivalent polysaccharide

*Corresponding author: Corwin A Robertson, Sanofi Pasteur, Swiftwater, Pennsylvania, USA, Tel: +1-570-957-0968; E-mail: corey.robertson@sanofipasteur.com

Received September 23, 2013; Accepted November 15, 2013; Published November 20, 2013

Citation: Robertson CA, Oster P, Johnson DR, Reinhardt A, Greenberg DP, et al. (2013) Prevention of Invasive Meningococcal Disease in the United States: Current State of the Art. J Vaccines Vaccin 4: 211. doi: 10.4172/2157-7560.1000211

Copyright: ( 2013 Robertson CA, et al. This is an open-access article distributed under the terms of the Creative Commons Attribution License, which permits unrestricted use, distribution, and reproduction in any medium, provided the original author and source are credited. 
vaccine (MPSV4; Menomune ${ }^{\circledR}$, Sanofi Pasteur), which demonstrates good vaccine effectiveness (VE) and an acceptable tolerability profile in most groups examined [16]. However, polysaccharide vaccines are poorly immunogenic in young children and do not induce immunologic memory [16,17]. Since the introduction of MPSV4, two quadrivalent conjugated meningococcal vaccines (MCV4) have been licensed in the United States [18,19]. Conjugated vaccines are effective across many age groups, produce a robust and anamnestic immune response and are associated with a reduction in IMD [17-23]. In addition, the impact on oropharyngeal carriage and transmission has been demonstrated for serogroups A, C, and Y [16,24,25] and is assumed for serogroup W. Meanwhile, the development of a vaccine against serogroup B has been challenging; the poor immunogenicity of the polysaccharide capsule is a consequence of its similarity to components of fetal and neural tissues [26,27]. Serogroup B vaccines composed of PorA-specific Outer Membrane Vesicles (OMV) have been used with success to control clonal outbreaks in Cuba and New Zealand $[28,29]$. Because such vaccines are directed against antigens specific to local serogroup B strains (serosubtypes), these vaccines would not be effective in continental countries, which have more diverse circulating serogroup B strains [28]. Currently, a serogroup B vaccine, combining the New Zealand OMV and several recombinant antigens, with potentially broader strain coverage, has been licensed in Europe but not in the United States.

\section{US Epidemiology}

\section{Overview}

In the United States, N. meningitidis is responsible for both sporadic and epidemic disease $[13,30]$. The annual incidence of meningococcal disease has varied from 0.5-1.5 cases per 100,000 since World War II [31]. In recent years, the overall rate of IMD in the general US population declined from 0.92 cases per 100,000 in 1998 [32,33] to 0.15 per 100,000 in 2010-a historic low [34].

In 2011, the most recent year for which data are available from the Active Bacterial Core surveillance (ABCs) program-an active surveillance system for invasive disease caused by selected bacterial pathogens-a total of 620 cases of IMD were estimated nationally, with 100 estimated fatalities (16\%) [7]. This represents $a \geq 3$-fold reduction from approximately a decade earlier, when national case and death estimates were 2200 and 300, respectively [35]. Although the precise reasons for the decline in IMD are unknown, changes in environmental, behavioral, microbial and host factors are suspected [33]. Immunization practices may have had an impact, specifically in pre-adolescents and adolescents for whom universal vaccination is recommended [36]. Among persons aged 11-19 years, the incidence of IMD caused by serogroups $\mathrm{C}, \mathrm{W}$ and $\mathrm{Y}$ decreased by approximately $50 \%$ in 2008 and 2009 compared with the rate observed during 19932005 [32].

ABCs data also reveal that the distribution of serogroups in the United States changes over time. In 2011, serogroup Y accounted for $36 \%$ of the 75 IMD cases detected by the system [7] (Figure 1). This is in contrast to the period 1989-1991 when serogroup Y caused only $2 \%$ of detected IMD cases [37] (Figure 1). Despite a decrease in relative prevalence from $45 \%$ in $1989-1991$ to $25 \%$ in 2011, serogroup C still remains a major cause of vaccine-preventable IMD [7,37]. These changes in serogroup distribution emerged over a period of approximately 2 decades. In the pre-World War II era, serogroup A was the cause of very large US epidemics [38], but in recent decades, few or no serogroup A
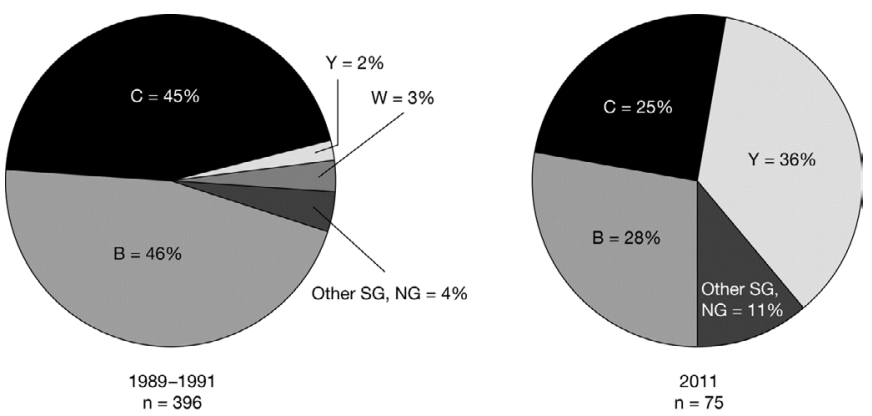

SG: serogroup; NG: nongroupable

Figure 1: Relative distribution of meningococcal serogroups over time in the US $[7,37]$.

cases have been reported [7]. Though the proportion of $\mathrm{W}$ disease has remained relatively low in the United States $[7,34,39,40]$, serogroup W has been responsible for recent outbreaks (including in the Western hemisphere) [41]. This observation suggests that serogroup $\mathrm{W}$ has the potential to become an increasing threat in the United States. Given the varying epidemiology of IMD, as well as increasing global travel, a prudent vaccination strategy from both the personal and the public health viewpoint is to provide broad rather than narrow serogroup coverage.

As noted, IMD incidence rates vary by age. The rates of IMD are highest in infants and very young children, decline throughout childhood, rise again in adolescence, decline during most of adulthood, but then begin rising again in the sixth and later decades of life (indeed, rates among those aged 65 years and older approach those seen in adolescents, likely due to declining immune competence) [3]. In young children, the higher rate of IMD may reflect less developed innate and specific immunity against $N$. meningitides [42,43]. Meningococcal carriage prevalence also varies with age and, interestingly, the prevalence is highest in adolescence and lowest in young children [44]. The increase in carriage prevalence from childhood to adolescence suggests that the disease peak in adolescents may be related to increased exposure in this population [44]. Consistent with this observation, there is an increased incidence of IMD in college students (typically aged 18-22 years), who tend to reside within dormitories, a known risk factor for both $N$. meningitidis carriage [45] and clinical disease [46-48]. This increased risk of IMD among dormitory residents was observed in the United States as early as the 1990s [47,49] and has also been observed in other countries. A significantly greater incidence of IMD was reported among UK university students versus age-matched nonstudents [48]. In 2011, a higher rate of vaccine-preventable IMD was observed in young adults aged $18-22$ years $(0.15$ per 100,000$)$ compared with adolescents aged 11-17 years $(0.03$ per 100,000$)$ [7]. The lower rate in the adolescents may also reflect changes in the 2005 Advisory Committee on Immunization Practices (ACIP) vaccination recommendations, as a comparison of the same age groups prior to the availability of MCV4 revealed that the incidence rate from these two cohorts was more similar (18-22 years: 0.26 per 100,000 vs. $11-17$ years: 0.18 per 100,000$)$ [50].

Prior to routine vaccination of recruits, military personnel, who commonly reside in congregate housing, also experienced a higher rate of IMD than the general population [51]. Likely risk factors include prolonged close contact with other soldiers, exposure to peers carrying serogroups not native to the soldier's home and deployment 
to areas where $N$. meningitidis is endemic. These risk factors prompted the US military to initiate immunization against meningococcal disease in 1971, a strategy that has proven highly successful. With the immunization campaign, the crude rate of disease declined by $94.2 \%$; rates were 23.6 cases per 100,000 person-years during 1964-1971 (before meningococcal vaccines of any kind were introduced) and 1.4 cases per 100,000 person-years during 1983-1998 (after introduction of polysaccharide vaccine) [52]. The US military's switch to MCV4 in February 2010 is expected to provide additional benefit through the reduction of bacterial carriage as has been observed previously [53], preventing importation of meningococci when soldiers return home.

To further control disease in at-risk groups such as adolescents, young adults and military personnel, ACIP made recommendations regarding the use of MCV4 after the vaccine's introduction in 2005 [54]. Since that time, the rate of vaccine-preventable disease in 11-17 year olds has declined six-fold $(0.18$ per 100,000 vs. 0.03 per 100,000$)[7,50]$, while a similar decline in disease incidence has not been observed in other cohorts [33]. Future vaccination recommendations should be developed with current epidemiologic trends in mind, targeting those who remain at increased risk for disease.

\section{Outbreak-related considerations}

Outbreaks account for fewer than 2\% of all US cases of IMD but are associated with a higher case-fatality rate than sporadic disease (21\% vs. $11 \%)$ [30]. Fortunately, outbreak-related disease is typically preventable with vaccines: during 1994-2002, 66.4\% of US outbreaks were attributable to serogroup C [30]. Coincident with the overall decline in meningococcal disease incidence, outbreaks have apparently declined in number and size in recent years. Reported IMD outbreaks in the United States are summarized in the Table 1.

One cause of IMD outbreaks is the return of travelers from areas of endemic disease, allowing the import of new serogroups. This is illustrated by the importation and spread of serogroup W. In 2000, three confirmed cases of serogroup $\mathrm{W}$ meningococcal disease appeared in New York City related to individuals returning from the Hajj in Saudi Arabia [65]. The affected individuals included a returning pilgrim, a household contact of a returning pilgrim and an individual who may have interacted with Hajj pilgrims 5 days before becoming ill [65]. The latter two cases demonstrate that $N$. meningitidis can be transmitted from travelers to the general population. Dull et al. further investigated the transmission of $N$. meningitidis in Hajj pilgrims, by assessing oropharyngeal carriage in this population prior to and after their travel to Saudi Arabia. In this study, none tested were identified as carriers before departing the United States; however, $1.3 \%$ of study participants had evidence of serogroup $\mathrm{W}$ carriage after returning to the United States [66]. Travel was also implicated in another outbreak of serogroup W disease in southeastern Florida from 2008-2009. Common exposures, social settings or other connections among patients could not be identified, but the dominant clonal complex observed was identical to the dominant type observed in a recent Argentina and Brazil outbreak, suggesting importation from South America [41]. Most recently, a nationwide increase inserogroup W disease was reported in Chile, with 83 confirmed cases and 22 deaths occurring from January 2012 through June 2013 [67]. These incidents highlight the need for continued surveillance as serogroups uncommon in the United States can easily be imported.

Although outbreaks-once detected-can be managed with reactive vaccination, this strategy is not ideal, as substantial disease can occur before mobilizing immunization efforts [47]. Chemoprophylaxis of close contacts is routine, but drug administration within 24 hours of meningococcal exposure is advised for optimum protection [4]. The issue of chemoprophylaxis is confounded by antibiotic-resistant $N$. meningitidis strains; fluoroquinolone-resistant $N$. meningitidis has been detected in Minnesota and North Dakota [68,69].

\section{Meningococcal vaccines in the United States and acip recommendations}

Beginning in 1969, meningococcal polysaccharide monovalent vaccines for serogroups $\mathrm{A}$ and $\mathrm{C}$ were introduced in the US based upon efficacy studies in human volunteers [70]. These vaccines were found to be effective and well tolerated in older children and adults. In children aged 18 monthsand younger, the monovalent meningococcal serogroup $\mathrm{C}$ polysaccharide vaccine was poorly immunogenic and, similar to monovalent A vaccine, provided relatively short-term protection. The persistence of immunity from both vaccines was 3-5 years. In 1981, the first quadrivalent polysaccharide meningococcal vaccine was approved for use in the United States [3]. MPSV4 consists of a lyophilized component of group-specific polysaccharide antigens from N. meningitidis serogroups A, C, Y and W. MPSV4 is approved for use in persons aged $\geq 2$ years [71]. The response to the vaccine is consistent with that to a T-cell-independent antigen: an age-dependent response, with poor immunity in children aged $<2$ years. MPSV4 use in US military personnel during 1983-1998 successfully prevented IMD, with a $40 \%$ reduction from the crude disease rate for the period 19791982, when bivalent (serogroups A and C) polysaccharide vaccine was in use [52].

\begin{tabular}{|c|c|c|c|c|c|c|}
\hline Location & Cohort affected & Cases, $\mathbf{n}$ & $\begin{array}{c}\text { Case-fatality rate, } \\
\%(n)\end{array}$ & Serogroup & Year & $\begin{array}{c}\text { Vaccination used as a } \\
\text { control measure }\end{array}$ \\
\hline New York, NY [55-57] & Men who have sex with men & $22^{*}$ & $32(7)$ & $\mathrm{C}$ & 2010-2013 & Yes \\
\hline $\begin{array}{l}\text { Crook County, OR } \\
{[58]}\end{array}$ & Aged $<25$ y & 6 & $0(0)$ & $\mathrm{C}$ & $2011-2012$ & Yes \\
\hline $\begin{array}{l}\text { Larimer County, CO } \\
\text { [59-61] }\end{array}$ & College students and adult hockey team & 9 & $56(5)$ & C & 2010 & Yes \\
\hline Rogers County, OK [62] & $\begin{array}{l}\text { Elementary school students (aged 6-8 y) plus } \\
\text { one high school student }\end{array}$ & 5 & $40(2)$ & $\mathrm{C}$ & 2010 & Yes \\
\hline $\begin{array}{l}\text { Southeastern, FL } \\
\text { [41] }\end{array}$ & Aged $1-84$ y & 14 & $29(4)$ & W & 2008-2009 & Not \\
\hline Brooklyn, NY [63] & Illicit drug users & 23 & $30(7)$ & $\mathrm{C}$ & $2005-2006$ & Yes \\
\hline Chicago, IL [64] & Men who have sex with men & 6 & $50(3)$ & $\mathrm{C}$ & 2003 & Yes \\
\hline
\end{tabular}

*A 23rd man from outside NYC who spent significant time in the NYC boroughs also became ill but later recovered †Broad age range and varied demographics made it difficult to identify whom to vaccinate

Table 1: Reported outbreaks of invasive meningococcal disease in the US, 2003-2013. 
As noted, college students have both high rates of $N$. meningitidis carriage [45] and clinical disease [46-48]. To prevent IMD in highrisk college students, ACIP recommended in 2000 that 1) healthcare providers should inform students and their parents of the increased risk of IMD and of the potential benefits of MPSV4 vaccination, 2) that colleges and universities should implement IMD educational programs, and 3) that MPSV4 should be readily available to students [72].

To enhance IMD prevention, public health officials called on industry to develop conjugate vaccines against $N$. meningitidis. Conjugation alters the immune response by inducing a T-celldependent response as opposed to the T-cell-independent (i.e., B-cell only) immune response. The former is more robust and longer lasting and leads to immune memory. Furthermore, conjugated vaccines can be used in children aged $<2$ years and may reduce $N$. meningitidis carriage [73]. In 2005, a meningococcal (groups A, C, Y and W) polysaccharide diphtheria toxoid conjugate vaccine (Menactra ${ }^{\circledR}$, Sanofi Pasteur; MenACWY-D) was licensed in the United States as a liquid formulation in a single vial [18]. The approval of MenACWY-D was based on studies comparing immunogenicity of a single MenACWY-D dose to a single MPSV4 dose, as assessed by postvaccination levels of complement-dependent serum bactericidal antibody (SBA).* A substantial increase in SBA was observed in individuals vaccinated with MenACWY-D [17,74-77].

In 2005, ACIP recommended routinely immunizing 11-12 year olds with one dose of Men ACWY-D during their yearly healthcare visit, and for adolescents older than 12 years, vaccination was recommended to occur before high school entry. Routine vaccination for those aged 11-55 years was also recommended if they were college freshman living in dormitories, microbiologists working with $N$. meningitidis, military recruits, travelers to IMD hyperendemic or epidemic areas or individuals with terminal complement deficiencies or asplenia [3] (Figure 2). Due to initial vaccine supply limitations, a catch-up program was not recommended [78].

In June 2007, ACIP revised its recommendation to include routine vaccination of all 11-18 year olds with one dose of Men ACWY-D at the earliest opportunity [78]. ACIP continued to recommend that 1112 year olds be routinely vaccinated [78] (Figure 2). Partly as a result of these recommendations, vaccination coverage increased substantially among adolescents. In 2008, 41.8\% of 13-17 year olds had received at least one dose of meningococcal vaccine; by 2012 , the percentage had increased to $74.0 \%[84,85]$. While MenACWY-D coverage did vary substantially by state following its introduction in 2005 [84], as previously mentioned, by $2008-2009$, the proportion of IMD attributable to serogroups $\mathrm{C}, \mathrm{W}$ and $\mathrm{Y}$ had decreased approximately $50 \%$ in persons aged 11-19 years compared with the rate observed during 1993-2005 [32]. Although these are encouraging trends, efforts to increase population vaccine coverage need to continue [21].

The VE of MenACWY-D was estimated by analyzing cases reported to both the $\mathrm{ABCs}$ and MeningNet, a passive surveillance system. Within 3-4 years, MenACWY-D VE was calculated to be 80$85 \%$ [22], despite the absence of a catch-up vaccination program and limited vaccine coverage among adolescents. This early VE estimate is similar to the efficacy measured for MPSV4 [22]. For comparison, a UK vaccine coverage campaign with meningococcal serogroup $\mathrm{C}$ conjugate vaccine (MCC) targeted both infants and those aged $<18$ years and achieved vaccine coverage rates exceeding $85 \%$ in most cohorts. The
VE of MCC used in this campaign was estimated to be $96 \%$ within 1 year of vaccination $[22,23]$.

In December 2007, the MenACWY-D indication was broadened to include children aged 2-10 years [20] based on results from a

2005: MenACWY-D licensed. Routine vaccination of adolescents with MenACWY-D first recommended

2006: Recommendation limited to cohorts of children entering high school or college and persons $11-55 \mathrm{y}$ of age at increased risk for meningococcal disease due to vaccine supply

2007: All adolescents $11-18$ y of age recommended for vaccination; children 2-10 y of age at increased risk for meningococcal disease recommended for vaccination

2009: Booster dose recommended for persons who remain at increased risk for meningococcal
disease, administered every $5 \mathrm{y}$; children who remain at risk and received primary dose at age $<7 \mathrm{y}$ recommended to receive booster dose $3 \mathrm{y}$ after their primary dose and then every $5 \mathrm{y}$ thereafter

2010: MenACWY-CRM licensed. Booster dose of MCV4 recommended for adolescents at age $16 \mathrm{y}$ and two-dose primary series for persons with certain immunocompromising conditions

2011: Two-dose primary series of MenACWY-D recommended for children 9-23 mo of age at increased risk for meningococcal disease

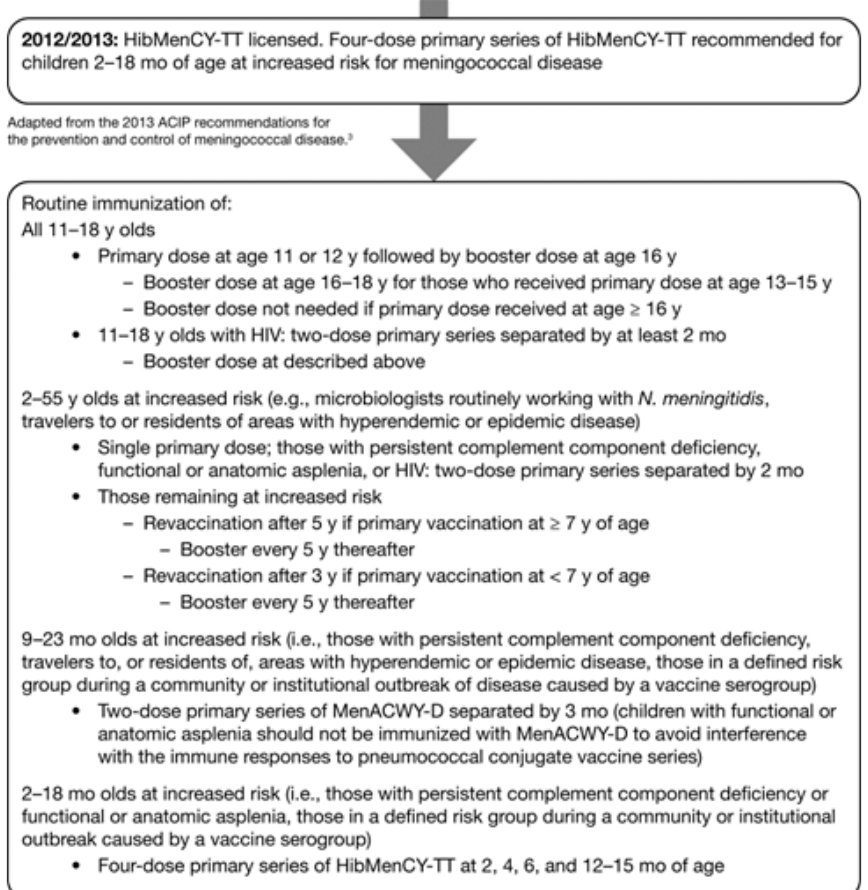

Figure 2: History of ACIP recommendations on the prevention of invasive meningococcal disease with meningococcal conjugate vaccine $[3,20,36,79$ 83].

*Complement is a necessary reagent for antibody-mediated killing of meningococc in serum bactericidal assays. Use of complement from either baby rabbit or human sources is considered acceptable by the US Food and Drug Administration as a basis of licensure of meningococcal vaccines among adolescents and adults, whereas human complement is preferred for studies among infants and young children. Of note, human complement is difficult to obtain and has highly variable activity from batch to batch [74-76]. In contrast, baby rabbit is more easily obtained and has been shown to produce consistent and reproducible results [76,77]. For US licensure purposes, serum bactericidal assay (SBA) titer levels presumed to provide protection against meningococcal disease are $\geq 1: 128$ for SBA using baby rabbit complement (rSBA) and $\geq 1: 8$ for SBA using human complement (hSBA). 
modified double-blinded, randomized, controlled study that found MenACWY-D was more immunogenic than MPSV4 [86]. However, ACIP advised against the routine immunization of all 2-10 year olds, instead recommending vaccination only for those at an increased risk of IMD [20,82] (Figure 2). This recommendation reflects current disease epidemiology (disease incidence in 2-10 year olds is lower than in infants and adolescents) and the notion that immunologic responses elicited in 2 year olds are unlikely to protect into adolescence-the next high-risk age period [82]. The 2007 recommendation also stated that vaccination with Men ACWY-D was preferable to MPSV4 and that for children aged 2-10 years previously vaccinated with a polysaccharide vaccine, a 3-year follow-up Men ACWY-D vaccination was recommended.

In 2010, the FDA approved a meningococcal (groups A, C, W and Y) oligosaccharide diphtheria $\mathrm{CRM}_{197}$ conjugate vaccine (MenACWYCRM; Menveo ${ }^{\circledR}$, Novartis). This vaccine was the second conjugate vaccine targeting serogroups $\mathrm{A}, \mathrm{C}, \mathrm{W}$ and $\mathrm{Y}$; collectively MenACWY-D and MenACWY-CRM are abbreviated as MCV4. MenACWY-CRM consists of meningococcal polysaccharides conjugated to a nontoxic mutated diphtheria toxin $\left(\mathrm{CRM}_{197}\right)$ [19]. The MenACWY-CRM presentation consists of two vials: a vial containing vaccine against serogroups $\mathrm{C}, \mathrm{W}$ and $\mathrm{Y}$ in a liquid formulation and a vial containing lyophilized vaccine against serogroup A. The vial contents are mixed before MenACWY-CRM administration [19]. Initially, MenACWYCRM was approved for individuals aged 11-55 years, but in 2011, the approved age range was extended to children aged 2-10 years and then to children as young as 2 months of age in $2013[19,80]$. The approval of MenACWY-CRM in persons 2 years- 55 yearswas based upon SBA responses in individuals vaccinated with either MenACWYCRM or MenACWY-D. Antibody responses after MenACWY-CRM vaccination were found to be noninferior to MenACWY-D vaccination for all serogroups except for serogroup A in children aged 2-10 years (the proportion with serogroup A hSBA titers $\geq 1: 8$ for MenACWYCRM vs. MenACWY-D were $72 \%$ vs. $77 \%$ for $2-5$ year olds and $77 \%$ vs. $83 \%$ for $6-10$ year olds) $[19,87]$. Follow-up in adolescents 22 months postvaccination demonstrated that those immunized with MenACWYCRM had higher hSBA titers than those who received MenACWY-D [88]. However, at 3 years postimmunization, the titer differences had waned and the proportions of adolescents with hSBA $\geq 1: 8$ against each of the four serogroups was similar for MenACWY-D and MenACWYCRM [80]. The clinical relevance of initially higher antibody titers is unclear [89]. Although VE data for MenACWY-CRM are not available, the vaccine has been shown to reduce carriage of serogroups $\mathrm{C}, \mathrm{W}$, and $\mathrm{Y}$ combined (reduction of 36\%) in English university students $\geq 2$ months after vaccination [25].

Analysis of 2006-2010 data demonstrated that the VE of MenACWY-D wanes over time. Based on this finding and antibody persistence data for both MenACWY-D and MenACWY-CRM, ACIP hypothesized that approximately $50 \%$ of individuals immunized at age 11-12 years would be unprotected beyond 5 years [36] (representing the high-risk period at age 16-21 years). Although it might appear that quadrivalent polysaccharide vaccines offer a similar duration of protection [36], it must be remembered that polysaccharide vaccines neither prime nor boost, whereas readministration of conjugate vaccine produces a strong anamnestic response [17]. In 2010, ACIP continued to recommend the routine immunization of 11-12 year olds with MCV4, but added the stipulation that a booster dose be administered at age 16 years [36] (Figure 2).
Of note, durability of immune response has been shown to be agedependent. In a study of MCCs used in the United Kingdom, follow-up rSBA titers were lower for those receiving the primary dose of MCC at age 6-9 years than those who received the primary dose at age 1015 years [90]. Another study found that among adolescents receiving MenACWY-D, 71-96\% had rSBA titers $\geq 1: 1283$ years post vaccination [90,91]. Among children initially immunized with MenACWY-D at age 2-3 years, $52-90 \%$ had $r S B A$ titers $\geq 1: 1282-3$ years post vaccination [92]. These findings prompted ACIP in 2009 to advise that individuals with prolonged increased risk of IMD be revaccinated after 5 years if immunized at age $\geq 7$ years and after 3 years if immunized at age 2-6 years (Figure 2). Since dormitory residence is a risk factor for invasive meningococcal disease [46-48], ACIP further recommended that adolescents vaccinated with MPSV $4 \geq 5$ years previously be immunized with MCV4 [83].

In 2010, ACIP refined its recommendation on the routine immunization of high-risk persons, advising that adolescents with human immunodeficiency virus (HIV) aged 11-18 years and persons aged 2-55 years with persistent complement component deficiency or functional or anatomic asplenia should receive two doses of MCV4, given 2 months apart [36] (Figure 2). Individuals deficient in either C7 or C9 are at 10,000- and 1400-fold greater risk, respectively, for IMD compared with those with an intact complement pathway $[93,94]$. Other associations between complement deficiencies and IMD risk and mortality have been reported [94]. A South African study found persons infected with HIV to be at an age-adjusted 11.3fold increased risk for IMD compared with HIV-negative individuals [95]. In a clinical study examining HIV-positive adolescents and young adults aged 11-24 years, a single dose of MenACWY-D resulted in $68 \%, 52 \%, 73 \%$ and $63 \%$ of participants achieving a $\geq 4$-fold increase in rSBA titers against serogroups A, C, W and Y, respectively [96]. Although these responses are not as robust as from individuals with fully functioning immune systems, a two-dose primary vaccination series with MenACWY-D has been shown to induce robust antibody responses in HIV-positive children aged 2-10 years (proportions with $\geq 4$-fold increase in rSBA titer: $88 \%, 80 \%, 100 \%$ and $84 \%$ for serogroups A, C, W and Y, respectively) [97]. Data regarding immune responses to MenACWY-CRM among HIV-positive individuals are not available. In 2011, the US indication for MenACWY-D was expanded to include children aged 9-23 months, allowing recommendation of vaccination against IMD in high-risk infants and toddlers. In 2012, a vaccine combining a bivalent conjugate meningococcal vaccine (serogroups $\mathrm{C}$ and $\mathrm{Y}$ ) with an Haemophilus influenzae type b (Hib) tetanus toxoid conjugate vaccine (HibMenCY-TT; MENHIBRIX ${ }^{\circledR}$, GlaxoSmithKline Biologicals) was approved in the United States for use in infants [98]. HibMenCY-TT is supplied as a lyophilized vaccine to be reconstituted with saline, and is administered as a four-dose series at 2, 4, 6 and 12-15 months of age [99]. Currently, ACIP recommends vaccinating infants 6 weeks through 18 months who are at increased risk of IMD [79] (Figure 2). HibMenCY-TT is the first meningococcal conjugate vaccine approved for use in persons aged 2-8 months [79]. In 2013, MenACWY-CRM, became the first quadrivalent meningococcal conjugate vaccine approved for use in this age group; it is administered as 4-dose series at 2, 4, 6 and 12 months of age [19]. As of this writing, ACIP recommendations regarding the use of MenACWY-CRM in infants are pending.

ACIP's recommendation to vaccinate infants and young children who are at high risk is based on US epidemiologic data and reflects the 
fact that presently the amount of vaccine-preventable IMD at this stage of life is low. Serogroup B is responsible for the majority of IMD cases, approximately $60 \%$, within the first year of life and it is not targeted by either MCV4 or HibMenCY-TT [3]. Meanwhile, serogroups C and $\mathrm{Y}$, which are included in current vaccines, are associated with disease incidence rates of 0.3 and 0.9 per 100,000 children aged $<1$ year (vs. 2.8 per 100,000 for serogroup B) [3]. Similarly, in children aged 2-4 years, the incidence rates are $0.1,0.1$ and 0.3 per 100,000 population for serogroups $\mathrm{C}, \mathrm{Y}$ and $\mathrm{B}$, respectively [3]. Mathematical models based on 2007-2009 epidemiologic data indicated that routine infant immunization would prevent 44 cases and 2-4 deaths per year, with a correspondingly high cost per quality-adjusted life year [32].

The relatively high cost of a universal infant recommendation is not the only barrier to its implementation. Such a recommendation is unlikely to contribute to herd immunity, as infants are not the primary reservoirs of $N$. meningitidis: adolescents and young adults are [100]. In the United Kingdom, a catch-up immunization program targeted persons aged $<18$ years with MCC. This program was associated with a reduction in carriage rates by $66 \%$ in students aged $15-17$ years [53] and a $67 \%$ reduction in the attack rate among the unvaccinated [101]. Thus, increased US adolescent coverage for both the primary and booster doses might improve herd immunity in that group, allowing for at least partial protection of those remaining unvaccinated, and could further impede bacterial transmission to infants and young children [32], thus augmenting vaccine effectiveness [101]. Lastly, the immaturity of the infant immune system requires more doses to achieve protective antibody titers than required later in life. A meta-analysis demonstrated that a single dose of an MCC administered to toddlers was as effective as a three-dose series administered during infancy [102].

\section{Discussion}

In summary, there are four vaccines licensed in the United States for the prevention of meningococcal disease. MPSV4 (Menomune ${ }^{\mathbb{R}}$; Sanofi Pasteur, Swiftwater, PA) is a quadrivalent polysaccharide vaccine approved for use in persons aged 2 years and older [71]. MenACWY-D (Menactra $^{\mathbb{}}$; Sanofi Pasteur, Swiftwater, PA) and MenACWY-CRM (Menveo ${ }^{\circledR}$; Novartis Vaccines and Diagnostics, Cambridge, MA) are quadrivalent conjugate vaccines; the former is approved for use in persons aged 9 months through 55 years and the latter is approved for use in persons aged 2 months through 55 years $[18,19]$. Finally, HibMenCY-TT (MenHibrix ${ }^{\mathbb{B}}$; GlaxoSmithKline, Rixensart, Belgium) is a conjugate vaccine that covers two meningococcal serogroups $(\mathrm{C}$ and $\mathrm{Y}$ ) and is approved for use in children aged 6 weeks through 18 months [98]. Several considerations regarding use of these vaccines in certain age groups follow.

Based on current epidemiology and vaccine characteristics, ACIP recommends routine immunization with a conjugate vaccine for infants and children who have certain risk factors for meningococcal disease. Due to the prevalence of serogroups A and W outside the United States, ACIP recommends use of MenACWY-D for infants and toddlers aged 9 months through 23 months who will travel to areas where meningococcal disease is hyperendemic or epidemic [3]. ACIP recommendations for use of MenACWY-CRM in infants at high risk for IMD are not yet available.ACIP does not recommend use of MenACWY-D in children aged $<2$ years of age with anatomic or functional asplenia who have not completed their pneumococcal conjugate vaccine series [81]. This guidance was based on pneumococcal disease being more important than IMD in this population and clinical trial data that suggest MenACWY-D may interfere with immune responses to pneumococcal conjugate vaccine [81] (although results of a functional [opsonophagocytic] assay suggest otherwise) [103]. Another noteworthy consideration regarding the use of meningococcal vaccines in childhood is that HibMenCY-TT cannot be concomitantly administered with other infant combination vaccines containing Hib antigens [3].

The ACIP recommendation to immunize 11-12 year olds with MCV4, followed by a booster dose at age 16 years, reflects the increased risk of disease in this cohort [36]. Partly as a result of vaccination, disease incidence has declined, but a broader herd immunity effect has not yet been confirmed, as disease remains stable in other age cohorts. The absence of an observed herd effect might be the result of still less than optimal vaccination coverage among adolescents and the lack of a broad-based catch-up program (e.g., from young children through young adults, as employed in the United Kingdom, Netherlands, and elsewhere). Given that the highest burden of preventable IMD occurs in adolescents and young adults, prevention efforts should continue to be focused on this group [32]. Moreover, adolescents are carriers of $N$. meningitidis, and the indirect benefits of herd immunity could be realized with improved coverage.

Although conjugate vaccines are not approved for use in persons aged 56 years and older, concerns about hyporesponsiveness following repeated doses of MPSV4 prompted ACIP to recommend that persons aged $\geq 56$ years previously vaccinated with a meningococcal vaccine and who remain at increased risk for IMD (e.g., microbiologists working with isolates of $N$. meningitidis, travelers and persons at risk as a result of a community outbreak) be revaccinated with MCV4 [3].

Despite advances in vaccine development, several challenges to IMD prevention in the United States remain. In infants and young children, effective prevention of IMD requires the ability to target serogroup B, since it is this serogroup that causes the greatest percentage of disease in this age group. Recently, a meningococcal group $B$ vaccine (rDNA, component, adsorbed) (4CMenB, Bexsero ${ }^{\circledR}$; Novartis Vaccines \& Diagnostics) with the potential to offer broad coverage against a number of serogroup B strains was approved for use in the European Union. The vaccine was found to be immunogenic when given as a four-dose series beginning at age 2 months [104], although the clinical effectiveness of $4 \mathrm{CMenB}$ has yet to be determined.

New MCV4 vaccines for infants are also on the horizon. In the European Union but not in the United States, a new meningococcal group A, C, W and Y conjugate vaccine (MenACWY-TT, Nimenrix ${ }^{\mathbb{B}}$; GlaxoSmithKline Biologicals) was approved for use in children aged 12 months and older [105]. A conjugate vaccine based on MenACWYTT, known as TetraMen-T (Sanofi Pasteur), is in phase II clinical trials. Other early pipeline vaccines are also under development, including vaccines protecting against all five clinically important serogroups (i.e., $\mathrm{A}, \mathrm{B}, \mathrm{C}, \mathrm{W}$, and $\mathrm{Y}$ ).

Comparing meningococcal vaccines is complicated by the fact that immunogenicity comparisons of the various meningococcal vaccineseither those that are currently available or on the horizon-cannot be done reliably on the basis of SBA titer results from disparate trials. The SBA requires fresh complement, but different lots of complementparticularly human complement-can give markedly different results. The issue is further complicated by lack of standardization in study design, procedures, data collection and analysis (e.g., differences in study populations, and the use or nonuse of concomitant vaccines, as well as differences in serology laboratories, assay methods and complement sources). 
Citation: Robertson CA, Oster P, Johnson DR, Reinhardt A, Greenberg DP, et al. (2013) Prevention of Invasive Meningococcal Disease in the United States: Current State of the Art. J Vaccines Vaccin 4: 211. doi: 10.4172/2157-7560.1000211

\section{Conclusions}

In summary, despite the current historically low incidence of IMD in the United States, many factors remain that could increase the threat of IMD: the emergence of antibiotic-resistant strains; outbreaks and resurges of IMD, including those caused by uncommon serogroups; suboptimal vaccine coverage; waning immunity; absence of herd immunity and, of course, the dynamic epidemiologic situation. It is necessary, while implementing current vaccination recommendations and programs, to remain vigilant for material changes in meningococcal epidemiology and to continue efforts to develop new and improved vaccines.

\section{Acknowledgments}

Medical writing support was provided by Shelley Lindley of PAREXEL. PAREXEL was funded by Sanofi Pasteur. Robert Lersch, of Sanofi Pasteur, reviewed and edited the manuscript.

\section{References}

1. World Health Organization (2012) Meningococcal meningitis. Fact sheet $\mathrm{N}^{\circ} 141$

2. American Academy of Pediatrics Committee on Infectious Diseases (2005) Prevention and control of meningococcal disease: recommendations for use of meningococcal vaccines in pediatric patients. Pediatrics 116: 496-505.

3. Cohn AC, MacNeil JR, Clark TA, Ortega-Sanchez IR, Briere EZ, et al. (2013) Prevention and control of meningococcal disease: recommendations of the Advisory Committee on Immunization Practices (ACIP). MMWR Recomm Rep 62: $1-28$

4. Granoff DM, Pelton S, Harrison LH (2012) Meningococcal vaccines. (6 $6^{\text {th }}$ Edn.), Elsevier/Saunders, Edinburgh.

5. Centers for Disease Control and Prevention (2011) Active Bacterial Core Surveillance Report, Emerging Infections Program Network Group A Streptococcus, 2011

6. Centers for Disease Control and Prevention (2011) Active Bacterial Core Surveillance Report, Emerging Infections Program Network, Group B Streptococcus, 2011

7. Centers for Disease Control and Prevention (2011) Active Bacterial Core Surveillance Report, Emerging Infections Program Network, Neisseria meningitidis, 2011.

8. Centers for Disease Control and Prevention (2011) Active Bacterial Core Surveillance Report, Emerging Infections Program Network, Streptococcus pneumoniae, 2011

9. Centers for Disease Control and Prevention (2013) Vaccines and Preventable Diseases: Factsheet: Meningococcal Diseases and Meningococcal Vaccines.

10. Thigpen MC, Whitney CG, Messonnier NE, Zell ER, Lynfield R, et al. (2011) Bacterial meningitis in the United States, 1998-2007. N Engl J Med 364: 20162025

11. Erickson LJ, De Wals P, McMahon J, Heim S (2001) Complications of meningococcal disease in college students. Clin Infect Dis 33: 737-739.

12. Harrison LH, Trotter CL, Ramsay ME (2009) Global epidemiology of meningococcal disease. Vaccine 27 Suppl 2: B51-63.

13. Rosenstein NE, Perkins BA, Stephens DS, Lefkowitz L, Cartter ML, et al. (1999) The changing epidemiology of meningococcal disease in the United States, 1992-1996. J Infect Dis 180: 1894-1901.

14. Vermont CL, de Groot R, Hazelzet JA (2002) Bench-to-bedside review: genetic influences on meningococcal disease. Crit Care 6: 60-65.

15. Murray RL, Britton J, Leonardi-Bee J (2012) Second hand smoke exposure and the risk of invasive meningococcal disease in children: systematic review and meta-analysis. BMC Public Health 12: 1062

16. Yogev R, Tan $T$ (2011) Meningococcal disease: the advances and challenges of meningococcal disease prevention. Hum Vaccin 7: 828-837.

17. Keyserling H, Papa T, Koranyi K, Ryall R, Bassily E, et al. (2005) Safety, immunogenicity, and immune memory of a novel meningococcal (groups A, C, $\mathrm{Y}$, and $\mathrm{W}-135)$ polysaccharide diphtheria toxoid conjugate vaccine (MCV-4) in healthy adolescents. Arch Pediatr Adolesc Med 159: 907-913.
18. Menactra ${ }^{\circledR}$ [prescribing information]. Swiftwater, PA, USA: Sanofi Pasteur Inc.

19. Menveo ${ }^{\circledR}$ [prescribing information]. Sovicille, Italy: Novartis Vaccines and Diagnostics.

20. Centers for Disease Control and Prevention (2007) Notice to Readers: Recommendation from the Advisory Committee on Immunization Practices (ACIP) for use of quadrivalent meningococcal conjugate vaccine (MCV4) in children aged 2-10 years at increased risk for invasive meningococcal disease. MMWR Morb Mortal Wkly Rep 56: 1265-1266.

21. Cohn A, Meyer S, MacNeil J (2012) Impact of quadrivalent meningococcal conjugate vaccine (Men ACWY) coverage on disease. Incidence in the United States, 2004-2010. Proceedings of the 18th International Pathogenic Neisseria Conference (IPNC).

22. Macneil JR, Cohn AC, Zell ER, Schmink S, Miller E, et al. (2011) Early estimate of the effectiveness of quadrivalent meningococcal conjugate vaccine. Pediatr Infect Dis J 30: 451-455.

23. Miller E, Salisbury D, Ramsay M (2001) Planning, registration, and implementation of an immunisation campaign against meningococca serogroup C disease in the UK: a success story. Vaccine 20 Suppl 1: S58-67.

24. Kristiansen PA, Diomandé F, Ba AK, Sanou I, Ouédraogo AS, et al. (2013) Impact of the serogroup A meningococcal conjugate vaccine, MenAfriVac, on carriage and herd immunity. Clin Infect Dis 56: 354-363.

25. Read RC, Baxter D, Chadwick DR, Faust SN, Finn A, et al. (2013) Impact of quadrivalent conjugate (MenACWY-CRM) and serogroup $B$ (4CMenB) meningococcal vaccines on meningococcal carriage in English university students. Proceedings of the 31st Annual Meeting of the European Society for Paediatric Infectious Diseases (ESPID).

26. Finne J, Bitter-Suermann D, Goridis C, Finne U (1987) An IgG monoclonal antibody to group $B$ meningococci cross-reacts with developmentally regulated polysialic acid units of glycoproteins in neural and extraneural tissues. J Immunol 138: 4402-4407.

27. Khatami A, Pollard AJ (2010) The epidemiology of meningococcal disease and the impact of vaccines. Expert Rev Vaccines 9: 285-298.

28. Panatto D, Amicizia D, Lai PL, Gasparini R (2011) Neisseria meningitidis $B$ vaccines. Expert Rev Vaccines 10: 1337-1351.

29. Sadarangani M, Pollard AJ (2010) Serogroup B meningococcal vaccines-an unfinished story. Lancet Infect Dis 10: 112-124.

30. Brooks R, Woods CW, Benjamin DK Jr, Rosenstein NE (2006) Increased case-fatality rate associated with outbreaks of Neisseria meningitidis infection compared with sporadic meningococcal disease, in the United States, 1994 2002. Clin Infect Dis 43: 49-54.

31. Harrison LH (2010) Epidemiological profile of meningococcal disease in the United States. Clin Infect Dis 50 Suppl 2: S37-44.

32. Centers for Disease Control and Prevention (2011) Advisory Committee on Immunization Practices. Summary Report, Atlanta, Georgia.

33. Cohn AC, MacNeil JR, Harrison LH, Hatcher C, Theodore J, et al. (2010) Changes in Neisseria meningitidis disease epidemiology in the United States, 1998-2007: implications for prevention of meningococcal disease. Clin Infect Dis 50: 184-191.

34. Centers for Disease Control and Prevention (2010) Active Bacterial Core Surveillance Report, Emerging Infections Program Network, Neisseria meningitidis, 2010

35. Centers for Disease Control and Prevention (2000) Active Bacterial Core Surveillance Report, Emerging Infections Program Network, Neisseria meningitidis, 2000

36. Centers for Disease Control and Prevention (CDC) (2011) Updated recommendations for use of meningococcal conjugate vaccines Advisory Committee on Immunization Practices (ACIP), 2010. MMWR Morb Mortal Wkly Rep 60: 72-76.

37. Jackson LA, Wenger JD (1993) Laboratory-based surveillance fo meningococcal disease in selected areas, United States, 1989-1991. MMWR CDC Surveill Summ 42: 21-30.

38. Bart A Schuurman IG, Achtman M, Caugant DA, Dankert J, et al. (1998) Randomly amplified polymorphic DNA genotyping of serogroup A meningococc yields results similar to those obtained by multilocus enzyme electrophoresis and reveals new genotypes. J Clin Microbiol 36: 1746-1749. 
Citation: Robertson CA, Oster P, Johnson DR, Reinhardt A, Greenberg DP, et al. (2013) Prevention of Invasive Meningococcal Disease in the United States: Current State of the Art. J Vaccines Vaccin 4: 211. doi: 10.4172/2157-7560.1000211

39. Centers for Disease Control and Prevention (2008) Active Bacterial Core Surveillance Report, Emerging Infections Program Network, Neisseria meningitidis, 2008

40. Centers for Disease Control and Prevention (2009) Active Bacterial Core Surveillance Report, Emerging Infections Program Network, Neisseria meningitidis, 2009

41. Doyle TJ, Mejia-Echeverry A, Fiorella P, Leguen F, Livengood J, et al. (2010) Cluster of serogroup W135 meningococci, southeastern Florida, 2008-2009. Emerg Infect Dis 16: 113-115.

42. Pollard AJ, Galassini R, Rouppe van der Voort EM, Hibberd M, Booy R, et al. (1999) Cellular immune responses to Neisseria meningitidis in children. Infect Immun 67: 2452-2463.

43. Pollard AJ, Galassini R, van der Voort EM, Booy R, Langford P, et al. (1999) Humoral immune responses to Neisseria meningitidis in children. Infect Immun 67: 2441-2451

44. Trotter CL, Gay NJ, Edmunds WJ (2006) The natural history of meningococcal carriage and disease. Epidemiol Infect 134: 556-566.

45. Neal KR, Nguyen-Van-Tam JS, Jeffrey N, Slack RC, Madeley RJ, et al. (2000) Changing carriage rate of Neisseria meningitidis among university students during the first week of term: cross sectional study. BMJ 320: 846-849.

46. Froeschle JE (1999) Meningococcal disease in college students. Clin Infect Dis 29: $215-216$.

47. Harrison LH, Dwyer DM, Maples CT, Billmann L (1999) Risk of meningococcal infection in college students. JAMA 281: 1906-1910.

48. Neal KR, Nguyen-Van-Tam J, Monk P, O’Brien SJ, Stuart J, et al. (1999) Invasive meningococcal disease among university undergraduates: association with universities providing relatively large amounts of catered hall accommodation. Epidemiol Infect 122: 351-357.

49. Harrison LH, Pass MA, Mendelsohn AB, Egri M, Rosenstein NE, et al. (2001) Invasive meningococcal disease in adolescents and young adults. JAMA 286: 694-699.

50. Centers for Disease Control and Prevention (2004) Active Bacterial Core Surveillance Report, Emerging Infections Program Network, Neisseria meningitidis, 2004

51. Keiser PB, Hamilton L, Broderick M (2011) U.S. military fatalities due to Neisseria meningitidis: case reports and historical perspective. Mil Med 176: 308-311.

52. Brundage JF, Ryan MA, Feighner BH, Erdtmann FJ (2002) Meningococcal disease among United States military service members in relation to routine uses of vaccines with different serogroup-specific components, 1964-1998. Clin Infect Dis 35: 1376-1381.

53. Maiden MC, Stuart JM; UK Meningococcal Carraige Group (2002) Carriage of serogroup C meningococci 1 year after meningococcal C conjugate polysaccharide vaccination. Lancet 359: 1829-1831.

54. Bilukha OO, Rosenstein N; National Center for Infectious Diseases, Centers for Disease Control and Prevention (CDC) (2005) Prevention and control of meningococcal disease. Recommendations of the Advisory Committee on Immunization Practices (ACIP). MMWR Recomm Rep 54: 1-21.

55. Centers for Disease Control and Prevention (CDC) (2013) Notes from the field: serogroup $C$ invasive meningococcal disease among men who have sex with men - New York City, 2010-2012. MMWR Morb Mortal Wkly Rep 61: 1048.

56. Health department issues new vaccination recommendations for men at greatest risk for contracting meningitis. The New York City Department of Health and Mental Hygiene.

57. Health advisory: expanded outbreak response meningococcal vaccine recommendations for men who have sex with men: update \#1. New York State Department of Health (NYSDOH) Division of Epidemiology.

58. Muriel DeLaVergne-Brown (2012) Crook County Meningococcal Press Release. Crook County Public Health Office.

59. Gibbons C (2011) Colorado urges vaccinating preteens against deadly meningococcal disease. Denver Post.

60. CSU student infected in new Larimer County meningococcal sepsis case. NBC News.
61. Meningococcal Disease Advisory Guidance for Colleges and Health-care Providers. Douglas County Government.

62. Centers for Disease Control and Prevention (CDC) (2012) Outbreak of meningococcal disease associated with an elementary school -- Oklahoma, March 2010. MMWR Morb Mortal Wkly Rep 61: 217-221.

63. Weiss D, Stern EJ, Zimmerman C, Bregman B, Yeung A, et al. (2009) Epidemiologic investigation and targeted vaccination initiative in response to an outbreak of meningococcal disease among illicit drug users in Brooklyn, New York. Clin Infect Dis 48: 894-901.

64. Schmink S, Watson JT, Coulson GB, Jones RC, Diaz PS, et al. (2007) Molecular epidemiology of Neisseria meningitidis isolates from an outbreak of meningococcal disease among men who have sex with men, Chicago, Illinois, 2003. J Clin Microbiol 45: 3768-3770.

65. Centers for Disease Control and Prevention (CDC) (2000) Serogroup W-135 meningococcal disease among travelers returning from Saudi Arabia--United States, 2000. MMWR Morb Mortal Wkly Rep 49: 345-346.

66. Dull PM, Abdelwahab J, Sacchi CT, Becker M, Noble CA, et al. (2005) Neisseria meningitidis serogroup W-135 carriage among US travelers to the $2001 \mathrm{Hajj}$. J Infect Dis 191: 33-39.

67. Ministerio de Salud Gobierno de Chile. Situación enfermedad meningocócica por serogrupo W-135 (desde 01/01/13 al 12/06/13) [in Spanish]. Ministerio de Salud Gobierno de Chile.

68. Centers for Disease Control and Prevention (CDC) (2008) Emergence of fluoroquinolone-resistant Neisseria meningitidis--Minnesota and North Dakota, 2007-2008. MMWR Morb Mortal Wkly Rep 57: 173-175.

69. Wu HM, Harcourt BH, Hatcher CP, Wei SC, Novak RT, et al. (2009) Emergence of ciprofloxacin-resistant Neisseria meningitidis in North America. N Engl J Med 360: 886-892.

70. Gotschlich EC, Goldschneider I, Artenstein MS (1969) Human immunity to the meningococcus. IV. Immunogenicity of group A and group C meningococcal polysaccharides in human volunteers. J Exp Med 129: 1367-1384.

71. Menomune ${ }^{\circledR}$ (2005) Meningococcal Polysaccharide Vaccine, Groups A, C, Y and W-135 Combined. Swiftwater, PA: Sanofi Pasteur Inc.

72. [No authors listed] (2000) Meningococcal disease and college students Recommendations of the Advisory Committee on Immunization Practices (ACIP). MMWR Recomm Rep 49: 13-20.

73. Lingappa JR, Rosenstein N, Zell ER, Shutt KA, Schuchat A, et al. (2001) Surveillance for meningococcal disease and strategies for use of conjugate meningococcal vaccines in the United States. Vaccine 19: 4566-4575.

74. Andrews N, Borrow R, Miller E (2003) Validation of serological correlate of protection for meningococcal $\mathrm{C}$ conjugate vaccine by using efficacy estimates from postlicensure surveillance in England. Clin Diagn Lab Immunol 10: 780 786

75. Borrow R, Balmer P, Miller E (2005) Meningococcal surrogates of protectionserum bactericidal antibody activity. Vaccine 23: 2222-2227.

76. Maslanka SE, Gheesling LL, Libutti DE, Donaldson KB, Harakeh HS, et al. (1997) Standardization and a multilaboratory comparison of Neisseria meningitidis serogroup $\mathrm{A}$ and $\mathrm{C}$ serum bactericidal assays. The Multilaboratory Study Group. Clin Diagn Lab Immunol 4: 156-167.

77. Lee LH (2004) FDA clinical briefing document for Aventis Pasteur Inc: Menactra ${ }^{\mathrm{TM}}$ : tetravalent meningococcal conjugate vaccine. Food and Drug Administration.

78. Centers for Disease Control and Prevention (CDC) Advisory Committee on Immunization Practices (2007) Revised recommendations of the Advisory Committee on Immunization Practices to Vaccinate all Persons Aged 11-18 Years with Meningococcal Conjugate Vaccine. MMWR Morb Mortal Wkly Rep 56: 794-795

79. Centers for Disease Control and Prevention (CDC) (2013) Infant meningococcal vaccination: Advisory Committee on Immunization Practices (ACIP) recommendations and rationale. MMWR Morb Mortal Wkly Rep 62: 52-54.

80. Centers for Disease Control and Prevention (CDC) (2011) Licensure of meningococcal conjugate vaccine for children aged 2 through 10 years and updated booster dose guidance for adolescents and other persons at increased risk for meningococcal disease--Advisory Committee on Immunization Practices (ACIP), 2011. MMWR Morb Mortal Wkly Rep 60: 1018-1019. 
Citation: Robertson CA, Oster P, Johnson DR, Reinhardt A, Greenberg DP, et al. (2013) Prevention of Invasive Meningococcal Disease in the United States: Current State of the Art. J Vaccines Vaccin 4: 211. doi: 10.4172/2157-7560.1000211

81. Centers for Disease Control and Prevention (2011) Recommendation of the Advisory Committee on Immunization Practices (ACIP) for use of quadrivalen meningococcal conjugate vaccine (MenACWY-D) among children aged 9 through 23 months at increased risk for invasive meningococcal disease. MMWR Morb Mortal Wkly Rep 60: 1391-1392.

82. Advisory Committee on Immunization Practices (ACIP) Centers for Disease Control and Prevention (CDC) (2008) Report from the Advisory Committee on Immunization Practices (ACIP): decision not to recommend routine vaccination of all children aged 2-10 years with quadrivalent meningococcal conjugate vaccine (MCV4). MMWR Morb Mortal Wkly Rep 57: 462-465.

83. Centers for Disease Control and Prevention (CDC) (2009) Updated recommendation from the Advisory Committee on Immunization Practices (ACIP) for revaccination of persons at prolonged increased risk for meningococcal disease. MMWR Morb Mortal Wkly Rep 58: 1042-1043.

84. Centers for Disease Control and Prevention (CDC) (2009) National, state, and local area vaccination coverage among adolescents aged 13-17 years--United States, 2008. MMWR Morb Mortal Wkly Rep 58: 997-1001.

85. Centers for Disease Control and Prevention (CDC) (2013) National and state vaccination coverage among adolescents aged 13-17 years--United States, 2012. MMWR Morb Mortal Wkly Rep 62: 685-693.

86. Pichichero M, Casey J, Blatter M, Rothstein E, Ryall R, et al. (2005) Comparative trial of the safety and immunogenicity of quadrivalent $(A, C, Y$, $\mathrm{W}$-135) meningococcal polysaccharide-diphtheria conjugate vaccine versus quadrivalent polysaccharide vaccine in two- to ten-year-old children. Pediatr Infect Dis J 24: 57-62.

87. Halperin SA, Gupta A, Jeanfreau R, Klein NP, Reisinger K, et al. (2010) Comparison of the safety and immunogenicity of an investigational and a licensed quadrivalent meningococcal conjugate vaccine in children 2-10 years of age. Vaccine 28: 7865-7872.

88. Gill CJ, Baxter R, Anemona A, Ciavarro G, Dull P (2010) Persistence of immune responses after a single dose of Novartis meningococcal serogroup $A$, C, W-135 and Y CRM-197 conjugate vaccine $\left(\right.$ Menveo $^{\mathbb{E}}$ ) or Menactra ${ }^{\mathbb{}}$ among healthy adolescents. Hum Vaccin 6: 881-887.

89. Centers for Disease Control and Prevention (CDC) (2010) Licensure of a meningococcal conjugate vaccine (Menveo) and guidance for use - Advisory Committee on Immunization Practices (ACIP), 2010. MMWR Morb Mortal Wkly Rep 59: 273.

90. Snape MD, Kelly DF, Lewis S, Banner C, Kibwana L, et al. (2008) Seroprotection against serogroup $\mathrm{C}$ meningococcal disease in adolescents in the United Kingdom: observational study. BMJ 336: 1487-1491.

91. Vu DM, Welsch JA, Zuno-Mitchell P, Dela Cruz JV, Granoff DM (2006) Antibody persistence 3 years after immunization of adolescents with quadrivalent meningococcal conjugate vaccine. J Infect Dis 193: 821-828.
92. Pichichero M, Papa T, Blatter M, Mitchell D, Kratz R, et al. (2006) Immune memory in children previously vaccinated with an experimental quadrivalent meningococcal polysaccharide diphtheria toxoid conjugate vaccine. Pediatr Infect Dis J 25: 995-1000.

93. Nagata M, Hara T, Aoki T, Mizuno Y, Akeda H, et al. (1989) Inherited deficiency of ninth component of complement: an increased risk of meningococcal meningitis. J Pediatr 114: 260-264.

94. Welsch JA, Ram S (2008) Factor H and neisserial pathogenesis. Vaccine 26 Suppl 8: 140-45.

95. Cohen C, Singh E, Wu HM, Martin S, de Gouveia L, et al. (2010) Increased incidence of meningococcal disease in HIV-infected individuals associated with higher case-fatality ratios in South Africa. AIDS 24: 1351-1360.

96. Siberry GK, Williams PL, Lujan-Zilbermann J, Warshaw MG, Spector SA et al. (2010) Phase I/II, open-label trial of safety and immunogenicity of meningococcal (groups A, C, Y, and W-135) polysaccharide diphtheria toxoid conjugate vaccine in human immunodeficiency virus-infected adolescents. Pediatr Infect Dis J 29: 391-396.

97. Siberry GK, Warshaw MG, Williams PL, Spector SA, Decker MD, et al. (2012) Safety and immunogenicity of quadrivalent meningococcal conjugate vaccine in 2- to 10-year-old human immunodeficiency virus-infected children. Pediatr Infect Dis J 31: 47-52.

98. Marion Gruber (2012) Meningococcal Groups $C$ and $Y$ and Haemophilus b Tetanus Toxoid Conjugate Vaccine. Rixensart, Belgium: GlaxoSmithKline.

99. MenHibrix (2012) Vaccines, Blood \& Biologics. U.S. Food and Drug Administration.

100. Caugant DA, Høiby EA, Magnus P, Scheel O, Hoel T, et al. (1994) Asymptomatic carriage of Neisseria meningitidis in a randomly sampled population. J Clin Microbiol 32: 323-330.

101. Ramsay ME, Andrews NJ, Trotter CL, Kaczmarski EB, Miller E (2003) Herd immunity from meningococcal serogroup $C$ conjugate vaccination in England: database analysis. BMJ 326: 365-366.

102. Pöllabauer EM, Petermann R, Ehrlich HJ (2005) Group C meningococcal polysaccharide-tetanus toxoid conjugate vaccine: a meta-analysis of immunogenicity, safety and posology. Hum Vaccin 1: 131-139.

103.Pina LM, Bassily E, Machmer A, Hou V, Reinhardt A (2012) Safety and immunogenicity of a quadrivalent meningococcal polysaccharide diphtheria toxoid conjugate vaccine in infants and toddlers: three multicenter phase III studies. Pediatr Infect Dis J 31: 1173-1183.

104. Bexsero European Public Assessment Report. European Medicines Agency. 105. Nimenrix European Public Assessment Report. European Medicines Agency. 